\title{
STAR-SHAPED COMPLEXES AND EHRHART POLYNOMIALS
}

\author{
TAKAYUKI HIBI
}

(Communicated by Wolmer V. Vasconcelos)

\begin{abstract}
We study Ehrhart polynomials of star-shaped triangulations of balls by means of Cohen-Macaulay rings and canonical modules.
\end{abstract}

A polyhedral complex $\Gamma$ in $\mathbb{R}^{N}$ is a finite set of convex polytopes in $\mathbb{R}^{N}$ such that

(1.1) if $\mathscr{P} \in \Gamma$ and $\mathscr{F}$ is a face of $\mathscr{P}$, then $\mathscr{F} \in \Gamma$, and

(1.2) if $\mathscr{P}, \mathscr{Q} \in \Gamma$, then $\mathscr{P} \cap \mathscr{Q}$ is a face of $\mathscr{P}$ and of $\mathscr{Q}$.

We are concerned with a polyhedral complex $\Gamma$ in $\mathbb{R}^{N}$ which satisfies the following conditions:

(2.1) every vertex $\alpha$ of $\mathscr{P} \in \Gamma$ has integer coordinates, i.e., $\alpha \in \mathbb{Z}^{N}$, and

(2.2) the underlying space $X:=\bigcup_{\mathscr{P} \in \Gamma} \mathscr{P}\left(\subset \mathbb{R}^{N}\right)$ of $\Gamma$ is homeomorphic to the $d$-ball.

Let $\partial X$ denote the boundary of $X$; thus $\partial X$ is homeomorphic to the $(d-$ 1)-sphere. Given an integer $n>0$, write $n X$ for $\{n \alpha ; \alpha \in X\}$ and define $i(X, n)$ to be $\#\left(n X \cap \mathbb{Z}^{N}\right)$, the cardinality of $n X \cap \mathbb{Z}^{N}$. In other words, $i(X, n)$ is equal to the number of rational points $\left(\alpha_{1}, \alpha_{2}, \ldots, \alpha_{N}\right) \in X$ with each $n \alpha_{i} \in \mathbb{Z}$. It is known that

(3.1) $i(X, n)$ is a polynomial in $n$ of degree $d$, called the Ehrhart polynomial of $X$,

(3.2) $i(X, 0)=1$, and

(3.3) $(-1)^{d} i(X,-n)=\#\left[n(X-\partial X) \cap \mathbb{Z}^{N}\right]$ for every $1 \leq n \in \mathbb{Z}$.

Define the sequence $\delta_{0}, \delta_{1}, \delta_{2}, \ldots$ of integers by the formula

$$
(1-\lambda)^{d+1}\left[1+\sum_{n=1}^{\infty} i(X, n) \lambda^{n}\right]=\sum_{i=0}^{\infty} \delta_{i} \lambda^{i} .
$$

Then

(4.1) $\delta_{0}=1$ and $\delta_{1}=\#\left(X \cap \mathbb{Z}^{N}\right)-(d+1)$,

(4.2) $\delta_{i}=0$ for each $i>d$, and

(4.3) $\delta_{d}=\#\left[(X-\partial X) \cap \mathbb{Z}^{N}\right]$.

We say that $\delta(X)=\left(\delta_{0}, \delta_{1}, \ldots, \delta_{d}\right)$ is the $\delta$-vector of $X$. We refer the reader to, e.g., [6, Chapter IX], for geometric proofs of the above fundamental results

Received by the editors June 18, 1993; this paper was presented in the meeting "Combinatorial Convexity and Algebraic Geometry" held at Mathematisches Forschungsinstitut Oberwolfach, March 28-April 3, 1993.

1991 Mathematics Subject Classification. Primary 13D40. 
due to Ehrhart. Note that, even though $X$ is not necessarily convex, the proofs in [6] are valid without modification since $X$ is homeomorphic to the $d$-ball.

Some algebraic technique ${ }^{1}$ is indispensable for the study of combinatorics on $\delta$-vectors. Fix a field $k$, and let $\xi_{1}, \xi_{2}, \ldots, \xi_{N}, t$ be (commutative) indeterminates over $k$. If $\alpha=\left(\alpha_{1}, \alpha_{2}, \ldots, \alpha_{N}\right) \in n X \cap \mathbb{Z}^{N}$, then we set $\xi^{\alpha} t^{n}=\xi_{1}^{\alpha_{1}} \xi_{2}^{\alpha_{2}} \ldots \xi_{N}^{\alpha_{N}} t^{n}$. We write $\left[A_{k}(\Gamma)\right]_{n}$ for the vector space spanned by all monomials $\xi^{\alpha} t^{n}$ with $\alpha \in n X \cap \mathbb{Z}^{N}$. Thus, in particular, $\operatorname{dim}_{k}\left[A_{k}(\Gamma)\right]_{n}=$ $i(X, n)$. Let $A_{k}(\Gamma)$ denote $\bigoplus_{n \geq 0}\left[A_{k}(\Gamma)\right]_{n}$ with $\left[A_{k}(\Gamma)\right]_{0}=k$, and define multiplication $\left(\xi^{\alpha} t^{n}\right)\left(\xi^{\beta} t^{m}\right)$ of monomials $\xi^{\alpha} t^{n}$ and $\xi^{\beta} t^{m}$ in $A_{k}(\Gamma)$ as follows: $\left(\xi^{\alpha} t^{n}\right)\left(\xi^{\beta} t^{m}\right)=\xi^{\alpha+\beta} t^{n+m}$ if there exists $\mathscr{P} \in \Gamma$ with $\alpha \in n \mathscr{P}$ and $\beta \in m \mathscr{P} ;\left(\xi^{\alpha} t^{n}\right)\left(\xi^{\beta} t^{m}\right)=0$ otherwise. Then $A_{k}(\Gamma)$ is a noetherian (i.e., finitely generated) graded algebra over $k$ and the Hilbert series $F\left(A_{k}(\Gamma), \lambda\right):=$ $\sum_{n=0}^{\infty} \operatorname{dim}_{k}\left[A_{k}(\Gamma)\right]_{n} \lambda^{n}$ is $\left(\delta_{0}+\delta_{1} \lambda+\delta_{2} \lambda^{2}+\cdots+\delta_{d} \lambda^{d}\right) /(1-\lambda)^{d+1}$. Let $\Omega\left(A_{k}(\Gamma)\right)=$ $\bigoplus_{n \geq 1}\left[\Omega\left(A_{k}(\Gamma)\right)\right]_{n}$ be the graded ideal of $A_{k}(\Gamma)$ which is generated by those monomials $\xi^{\alpha} t^{n}$ such that $0<n \in \mathbb{Z}$ and $\alpha \in n(X-\partial X) \cap \mathbb{Z}^{N}$. Since $X$ is homeomorphic to the $d$-ball, $A_{k}(\Gamma)$ is Cohen-Macaulay [10, Lemma 4.6]. Thus, a well-known technique of commutative algebra enables us to obtain $\delta(X) \geq 0$, i.e., each $\delta_{i} \geq 0$ (cf. Stanley [8]). On the other hand, the same technique as in the proof of [2, Theorem (5.6.1)] enables us to show that $\Omega\left(A_{k}(\Gamma)\right)$ is the canonical module of $A_{k}(\Gamma)$.

We say that $X$ is "star-shaped" with respect to a point $\alpha \in X-\partial X$ if $t \alpha+(1-t) \beta \in X-\partial X$ for every point $\beta \in X$ and for each real number $0<t<1$.

Theorem. We employ the same notation as used above. Suppose that the set $(X-\partial X) \cap \mathbb{Z}^{N}$ is nonempty and that the underlying space $X$ is star-shaped with respect to some $v_{1} \in(X-\partial X) \cap \mathbb{Z}^{N}$. Then the $\delta$-vector $\delta(X)=\left(\delta_{0}, \delta_{1}, \ldots, \delta_{d}\right)$ of $X$ satisfies the linear inequalities as follows:

$$
\begin{aligned}
& \delta_{0}+\delta_{1}+\cdots+\delta_{i} \leq \delta_{d}+\delta_{d-1}+\cdots+\delta_{d-i}, \\
& \delta_{1} \leq \delta_{i}, \quad 2 \leq i<d .
\end{aligned}
$$

Sketch of proof. First, recall that a simplicial complex in $\mathbb{R}^{N}$ is a polyhedral complex $\Delta$ in $\mathbb{R}^{N}$ such that every convex polytope belonging to $\Delta$ is a simplex in $\mathbb{R}^{N}$. Fix an arbitrary simplicial complex $\Delta(0)$ in $\mathbb{R}^{N}$ with the vertex set $\partial X \cap \mathbb{Z}^{N}$ whose underlying space is the boundary $\partial X$ of $X$. Since $X$ is starshaped with respect to $v_{1} \in(X-\partial X) \cap \mathbb{Z}^{N}$, we can define the cone $\Delta(1)$ over $\Delta(0)$ with apex $v_{1}$, i.e., $\Delta(1)$ is the simplicial complex in $\mathbb{R}^{N}$ which consists of those simplices $\sigma$ such that either $\sigma \in \Delta(0)$ or $\sigma$ is the convex hull of $\tau \cup\left\{v_{1}\right\}$ in $\mathbb{R}^{N}$ for some $\tau \in \Delta(0)$. The vertex set of $\Delta(1)$ is $\left(\partial X \cap \mathbb{Z}^{N}\right) \cup\left\{v_{1}\right\}$ and the underlying space of $\Delta(1)$ is $X$. Let $(X-\partial X) \cap \mathbb{Z}^{N}=\left\{v_{1}, v_{2}, \ldots, v_{\ell}\right\}$ and, for each $2 \leq j \leq \ell$, construct a simplicial complex $\Delta(j)$ with the vertex set $\left(\partial X \cap \mathbb{Z}^{N}\right) \cup\left\{v_{1}, v_{2}, \ldots, v_{j}\right\}$ and with the underlying space $X$ by the same way as in [7]. We write $\Delta$ for $\Delta(\ell)$. Then the element $\theta=\xi^{v_{1}} t+$ $\xi^{v_{2}} t+\cdots+\xi^{v_{\ell} t}$ of $\left[\Omega\left(A_{k}(\Delta)\right)\right]_{1}$ is a nonzero divisor on $A_{k}(\Delta)$. Hence, it follows from a standard technique of commutative algebra [11] (see also [4]) that $\sum_{0 \leq j \leq i} \delta_{j} \leq \sum_{0<j \leq i} \delta_{d-j}$ for every $0 \leq i \leq[d / 2]$. On the other hand, let $h(\Delta)=\left(h_{0}, h_{1}, \ldots, h_{d}, 0\right)$ be the $h$-vector (e.g., [9]) of the simplicial complex

\footnotetext{
${ }^{1}$ We refer to, e.g., [6, Chapter IV] for "Commutative Algebra for Combinatorialists".
} 
$\Delta$. Then $h_{1} \leq h_{i}$ for each $2 \leq i<d$ (cf. [7]). Also, $h_{1}=\delta_{1}$. Since $h_{i} \leq \delta_{i}$, $0 \leq i \leq d$, by [1], we have $\delta_{1} \leq \delta_{i}$ for each $2 \leq i<d$ as desired. Q.E.D.

Remark. (a) In the above sketch of proof, let $A_{k}(\Delta)^{*}$ denote the graded subalgebra of $A_{k}(\Delta)$ generated by $\left[A_{k}(\Delta)\right]_{1}$ over $k$. Then $A_{k}(\Delta)^{*}$ coincides with the Stanley-Reisner ring [9] of the simplicial complex $\Delta$. Thus $A_{k}(\Delta)^{*}$ is CohenMacaulay with the Hilbert series

$$
F\left(A_{k}(\Delta)^{*}, \lambda\right)=\left(h_{0}+h_{1} \lambda+h_{2} \lambda^{2}+\cdots+h_{d} \lambda^{d}\right) /(1-\lambda)^{d+1} .
$$

Moreover, $A_{k}(\Delta)$ is finitely generated as a module over $A_{k}(\Delta)^{*}$.

(b) By the similar method as in [3, Theorem (1.3)], without the hypothesis that $(X-\partial X) \cap \mathbb{Z}^{N}$ is nonempty and $X$ is star-shaped, we can prove that the $\delta$-vector $\delta(X)=\left(\delta_{0}, \delta_{1}, \ldots, \delta_{d}\right)$ of $X$ satisfies the linear inequality

$$
\delta_{d}+\delta_{d-1}+\cdots+\delta_{d-i} \leq \delta_{0}+\delta_{1}+\cdots+\delta_{i}+\delta_{i+1}
$$

for every $0 \leq i \leq[(d-1) / 2]$.

Example. Let $N=d=3$ and $X=\mathscr{P} \cup \mathscr{Q}$, where $\mathscr{P} \subset \mathbb{R}^{3}$ (resp. $\mathscr{Q} \subset \mathbb{R}^{3}$ ) is the tetrahedron with the vertices $(1,0,0),(0,1,0),(0,0,1),(-1,-1,-1)$ (resp. $(1,0,0),(0,1,0),(0,0,1),(1,1,0))$. Then $(X-\partial X) \cap \mathbb{Z}^{3}=$ $\{(0,0,0)\}$ and $X$ is not star-shaped with respect to $(0,0,0)$. However, $X$ is star-shaped with respect to, e.g., $(1 / 3,1 / 3,1 / 3)$. We have $\delta(X)=(1,2,1,1)$ which fails to satisfy (5.1) for $i=1$ and (5.2) for $i=2$.

Corollary $[3,7,11]$. Let $\mathscr{P} \subset \mathbb{R}^{N}$ be an integral convex polytope of dimension $d$, and suppose that $(\mathscr{P}-\partial \mathscr{P}) \cap \mathbb{Z}^{N}$ is nonempty. Then the $\delta$-vector $\delta(\mathscr{P})=$ $\left(\delta_{0}, \delta_{1}, \ldots, \delta_{d}\right)$ of $\mathscr{P}$ satisfies the following linear inequalities:

(6.1) $\delta_{0}+\delta_{1}+\cdots+\delta_{i} \leq \delta_{d}+\delta_{d-1}+\cdots+\delta_{d-i}, \quad 0 \leq i \leq[d / 2]$;

(6.2) $\delta_{d}+\delta_{d-1}+\cdots+\delta_{d-i} \leq \delta_{0}+\delta_{1}+\cdots+\delta_{i}+\delta_{i+1}, \quad 0 \leq i \leq[(d-1) / 2]$;

(6.3) $\delta_{1} \leq \delta_{i}, \quad 2 \leq i<d$.

We conclude the paper with a remark about the question when $A_{k}(\Gamma)$ is Gorenstein. For a while, we assume that $N=d$ and the origin of $\mathbb{R}^{d}$ is contained in the interior of $X$. We say that $\delta(X)=\left(\delta_{0}, \delta_{1}, \ldots, \delta_{d}\right)$ is symmetric if $\delta_{i}=\delta_{d-i}$ for every $0 \leq i \leq d$. It follows from, e.g., [5] that $X$ is star-shaped with respect to the origin if $\delta(X)$ is symmetric. On the other hand, $\delta(X)$ is symmetric if and only if there exists a polyhedral complex $\Gamma$ in $\mathbb{R}^{d}$ with the underlying space $X$ such that $A_{k}(\Gamma)$ is Gorenstein, i.e., the canonical module $\Omega\left(A_{k}(\Gamma)\right)$ of $A_{k}(\Gamma)$ is generated by a single element of $A_{k}(\Gamma)$.

\section{REFERENCES}

1. U. Betke and P. McMullen, Lattice points in lattice polytopes, Monatsh. Math. 99 (1985), 253-265.

2. W. Bruns and J. Herzog, Cohen-Macaulay rings, Cambridge Univ. Press, Cambridge, New York, and Sydney, 1993.

3. T. Hibi, Ehrhart polynomials of convex polytopes, $h$-vectors of simplicial complexes, and nonsingular projective toric varieties, Discrete and Computational Geometry (J. E. Goodman et al., eds.), DIMACS Ser. Discrete Math. Theoret. Comput. Sci., vol. 6, Amer. Math. Soc., Providence, RI, 1991, pp. 165-177.

4. ley-Reisner rings of distributive lattices, Pacific J. Math. 154 (1992), 253-264. 
5. —_ Dual polytopes of rational convex polytopes, Combinatorica 12 (1992), 237-240.

6. _ Algebraic combinatorics on convex polytopes, Carslaw, Sydney, 1992.

7. $\ldots$ A lower bound theorem for Ehrhart polynomials of convex polytopes, Adv. Math. 105 (1994), 162-165.

8. R. Stanley, Decompositions of rational convex polytopes, Ann. Discrete Math., vol. 6, NorthHolland, Amsterdam, 1980, pp. 333-342.

9. $\longrightarrow$, Combinatorics and commutative algebra, Birkhäuser, Boston, Basel, and Stuttgart, 1983.

10. - Generalized h-vectors, intersection cohomology of toric varieties, and related results, Commutative Algebra and Combinatorics (M. Nagata and H. Matsumura, eds.), Adv. Stud. Pure Math., vol. 11, North-Holland, Amsterdam, 1987, pp. 187-213.

11. 73 (1991), 307-314.

Department of Mathematics, Faculty of Science, Hokkaido University, Kita-KU, SaPPORO 060, JAPAN

E-mail address: hibi@math.hokudai.ac.jp 